\title{
Longitudinal genome-wide methylation study of Roux-en-Y gastric bypass patients reveals novel CpG sites associated with essential hypertension
}

\author{
Adrian E. Boström ${ }^{1 *}$, Jessica Mwinyi ${ }^{1}$, Sarah Voisin ${ }^{1}$, Wenting Wu$^{2}$, Bernd Schultes ${ }^{3}$, Kang Zhang ${ }^{2}$
} and Helgi B. Schiöth ${ }^{1}$

\begin{abstract}
Background: Essential hypertension is a significant risk factor for cardiovascular diseases. Emerging research suggests a role of DNA methylation in blood pressure physiology. We aimed to investigate epigenetic associations of promoter related $\mathrm{CpG}$ sites to essential hypertension in a genome-wide methylation approach.

Methods: The genome-wide methylation pattern in whole blood was measured in 11 obese patients before and six months after Roux-en-Y gastric bypass surgery using the Illumina $450 \mathrm{k}$ beadchip. CpG sites located within $1500 \mathrm{bp}$ of the transcriptional start site of adjacent genes were included in our study, resulting in 124199 probes investigated in the subsequent analysis. Percent changes in methylation states and SBP measured before and six months after surgery were calculated. These parameters were correlated to each other using the Spearman's rank correlation method (Edgeworth series approximation). To further investigate the detected relationship between candidate CpG sites and systolic blood pressure levels, binary logistic regression analyses were performed in a larger and independent cohort of 539 individuals aged 19-101 years to elucidate a relationship between EH and the methylation state in candidate $\mathrm{CpG}$ sites.
\end{abstract}

Results: We identified 24 promoter associated CpG sites that correlated with change in SBP after RYGB surgery $\left(p<10^{-16}\right)$. Two of these CpG loci (cg00875989, cg09134341) were significantly hypomethylated in dependency of EH $\left(p<10^{-03}\right)$. These results were independent of age, BMl, ethnicity and sex.

Conclusions: The identification of these novel $\mathrm{CpG}$ sites may contribute to a further understanding of the epigenetic regulatory mechanisms underlying the development of essential hypertension.

Keywords: Essential hypertension, Epigenome-wide association study, Whole methylome analysis, Bariatric surgery, EHMT2, FUSSEL18, SKOR2, Corl2

\section{Background}

Hypertension (HT) is the leading risk factor for mortality worldwide and is ranked third as a cause of disability-adjusted life-years. Today, nearly one billion people suffer from HT, making it an important publichealth issue [1]. The high prevalence of HT and its identification as a major risk factor for secondarily occurring

\footnotetext{
* Correspondence: adrian.bostrom@neuro.uu.se

${ }^{1}$ Department of Neuroscience, Functional Pharmacology, Uppsala University, BMC, Box 59375124 Uppsala, Sweden

Full list of author information is available at the end of the article
}

diseases of the cardiovascular system and the kidneys underlines the necessity to study the pathophysiological mechanisms and molecular biological susceptibility factors underlying the development of this disease.

The heritability of $\mathrm{HT}$ is estimated to range between 40 and $60 \%$ [2]. Besides the well-established link between genetic and non-genetic factors (such as e.g. obesity, alcohol or salt intake) in the pathogenesis of HT, there is increasing evidence that epigenetic mechanisms might be able to contribute to the development of HT [3]. Especially the missing heritability conundrum of HT 
combined with its late onset and progressive nature point to epigenetics as a potential modulator of blood pressure (BP). Epigenetic patterns are potentially reversible and are influenced by nutritional-environmental factors as well as gene-environment interactions. These factors have been shown to play an important role in the development of complex multifactorial diseases, such as those affecting the cardiovascular system [3]. An increasing number of studies suggest a significant role of epigenetic modifications in the progression of EH [4-6].

An elementary pillar in antihypertensive therapy is the reduction of weight. It has been shown that a modest weight loss by $10 \%$ already lowers the BP significantly $[7,8]$. Surgical interventions such as Roux-en-Y gastric bypass (RYGB) surgery are the most efficient therapeutic options for a significant reduction of weight and an improvement of associated cardiovascular and metabolic co-morbidities today [9]. The reduction in BP typically seen after surgery has been suggested to be an effect of both caloric restriction and weight loss $[10,11]$.

Age significantly impacts the pathogenesis of EH [12] and different sets of genes control BP regulation at different ages [13]. Furthermore, men have a significantly higher risk to develop cardiovascular disease than agematched premenopausal women [14]. In addition to disease risk alterations due to age, body weight and gender, the prevalence of $\mathrm{EH}$ varies in dependency of ethnic origin. So show individuals of Hispanic origin significantly lower BP levels than white individuals [15]. Inflammatory processes have also been shown to play a significant role in the pathophysiology of $\mathrm{EH}$, whereby the relative contribution of inflammation to BP variability remains to be fully clarified [16].

To date, very few studies have investigated the association between DNA methylation and BP levels in a genome-wide study approach. In this study, we perform a whole methylome analysis in obese patients undergoing RYGB surgery to examine whether and to what extent changes in DNA methylation patterns are associated with changes in systolic blood pressure (SBP). Newly identified candidate CpG loci are further investigated and confirmed as risk loci for $\mathrm{EH}$ in a targeted analysis performed in a large cohort of 539 individuals.

\section{Methods}

\section{Characterization of the discovery group and validation cohort}

\section{Study population of the discovery dataset}

Samples used in this study have been collected in the frame of a study that has been previously published [17]. The study population included twelve obese patients, who underwent bariatric surgery at the Klinik Stephanshorn in
St. Gallen, in 2009-2011. All subjects were eligible for bariatric surgery by international guidelines [18] and gave their written informed consent to participating in the study. In 2008 and prior to enrollment, the canton St. Gallen ethics committee approved the study.

Subjects were fasting at the time of blood sampling for whole methylome determination and measurements. Blood was drawn according to standard procedures and $\mathrm{BP}$ was measured one time after an overnight fast, in a sitting position, after $5 \mathrm{~min}$ of relaxation, before and six months after surgery. One patient suffered from a known genetic disorder and was not eligible for further analysis, resulting in a sample size of 11 individuals.

The surgical procedure was performed by connecting a 20-30 ml large gastric pouch from the upper stomach to the proximal jejunum, thereby considerably limiting the gastric residence time of nutrients. Furthermore, an anastomosis of the biliopancreatic limb to the jejunum was established $150 \mathrm{~cm}$ distal from the pouch-jejunal connection.

\section{Study population of the validation dataset}

Data for the validation study cohort were obtained from publicly available sources (ArrayExpress databases, accession number E-GEOD-40279). This cohort was composed of two different ethnic groups, comprising 426 Caucasians and 230 individuals of Mexican origin, aged 19 to 101 years old [19]. BMI was measured for all subjects. Information on blood pressure status (EH or normotensive) was provided to us by Zhang et al., and was available for 540 individuals. Information on intake of any anti-hypertensive medications, DBP or SBP, was not available. One patient was described as having 'borderline' $\mathrm{EH}$, and was therefore excluded in our analysis, resulting in a sample size of 539 observations.

\section{Methylation profiling}

Extraction of genomic DNA was performed by the phenol-chloroform method [20], after which it was bisulfite converted using the EZ DNA Methylation - GoldTM kit (ZymoResearch, USA). The bisulfite converted DNA was hybridized to the Illumina $450 \mathrm{k}$ methylation chip (Illumina, San Diego, CA, USA) to measure DNA methylation in 485577 individual CpG sites. In the discovery group, DNA obtained from blood samples taken from the same individual before and six months after surgery were hybridized to the same physical chip. The array was imaged using the Illumina iScan system (Illumina, San Diego, CA, USA), determining the percentile methylation for each $\mathrm{CpG}$ site across the study groups.

\section{CpG site annotation}

We used the expanded annotation table provided by Price et al. [21] to define the associated gene, distance to the 
closest TSS and the surrounding CpG density for each investigated $\mathrm{CpG}$ site. Probes are located in either a highdensity islands (HC), intermediate-density islands (IC) or in non-islands. The local $\mathrm{CpG}$ density has been shown to influence the role of methylated cytosines, whereby methylation influences the transcriptional efficacy stronger in high-density islands as compared to non-islands [22]. To ensure that variation in methylation reflect expression levels, the analysis was limited to CpG-sites located within 1500 base pairs up and upstream of the transcriptional start site. Wagner et al. revealed that the relationship between DNA methylation and gene expression is closely related within this region [23].

\section{Data processing}

To quantify methylation levels of $\mathrm{CpG}$ sites, raw data were transformed into $\beta$-values using the GenomeStudio software (Illumina, San Diego, CA, USA). Further data processing was performed using the $\mathrm{R}$ package Illumina Methylation Analyzer (IMA). CpG sites were excluded if located on sex determining chromosomes, covering known SNP loci or if $75 \%$ of subjects exhibited a detection $p$-value greater than $5 \times 10-05$.

\section{Adjustment of type I and type II probes and removal of batch effects}

CpG sites of the Illumina chip belong to type I or type II probes, which significantly differ in distribution and dynamic range of DNA methylation. As this design may bias downstream analyses, methylation data was further adjusted by peak correction and quantile normalization to adjust for probe type differences. The ComBat function [24] was used to adjust for batch effects.

After all steps of data processing procedures were performed, 124199 probes remained to be included in the subsequent analysis.

\section{Statistical analysis}

All statistical analyses were perfomed using R statistics, version 3.0.2.

'Hmisc' package of $\mathrm{R}$ statistic was used for discovery group analysis. When sample sizes exceed nine observations, as was the case in our study, this package by default uses algorithm AS 89 and an Edgeworth series approximation to calculate correlation coefficients and $p$-values for Spearman's rank correlation coefficients. We applied this method of analysis to our data. Percentage change in methylation states and SBP were calculated, measured before and six months after RYGB surgery. These parameters were correlated in using the Spearman's rank correlation method, resulting in 124199 individual correlation analyses. $P$-values were adjusted for multiple testing using Bonferroni correction method [25]. Adjusted $p$-values $<0.05$ were considered significant. To exclude potential confounding effects of age, BMI and levels of fasting plasma glucose, the same method of analysis was performed, correlating percentile changes in methylation states to these variables. Paired t-tests were performed to evaluate changes in continuous variables independently from each other, e.g. the methylation state of a CpG site before and six months after surgery.

In a second step, we aimed to further investigate the association of changed methylation patterns in candidate CpG sites with EH in a second cohort (verification cohort). For this purpose, binary logistic regressions of $\mathrm{EH}$ to the methylation states were performed, accounting for age, BMI, ethnicity and sex as co-variables.

\section{Results}

Behavior of clinical outcome variables in the discovery group and verification cohort

In the discovery group, comprising patients who underwent bariatric surgery, we initially aimed to identify candidate CpG sites, in which modifications of the epigenetic profile are associated with changes in SBP levels after surgical intervention. As expected, patients reduced their BMI by $24 \%$ six months after surgery. Furthermore, diastolic blood pressure (DBP) and SBP levels were significantly lower after the operation (Table 1).

CpG sites that correlated with SBP in the discovery group were further investigated for an association with $\mathrm{EH}$ in the verification cohort. This cohort comprised 539 subjects of Caucasian or Hispanic descent, aged 19 to 101 years and subgrouped into $\mathrm{EH}$ or normotensive individuals. While no significant differences with regard to ethnicity were observed in the hypertensive group, Hispanics were overrepresented in the normotensive study arm. In the hypertensive study arm white subjects were older than subjects of Hispanic origin. Furthermore, the individuals showed a significantly higher age and BMI in the hypertensive group as compared to the individuals in the normotensive study arm (Table 1).

\section{Methylation changes at distinct CpG sites correlate with the change in SBP in obese patients before and six months after RYGB surgery}

We performed a genome-wide methylation analysis in obese patients before and 6 months after RYGB surgery and compared the global methylation change with changes in SBP before and after the intervention. In case of $24 \mathrm{CpG}$ sites changes in methylation significantly correlated with the percentile change in SBP six months after RYGB surgery (Table 2). The changed methylation states of these CpG sites did not correlate with age, BMI or DBP, underlining the specificity of the observed associations. Methylation changes of four of these probes correlated also significantly with fasting plasma glucose 
Table 1 Characteristics of subjects included into the study

\begin{tabular}{|c|c|c|c|}
\hline \multirow[b]{2}{*}{ Parameter } & \multirow[t]{2}{*}{ Discovery group ${ }^{a}$} & \multicolumn{2}{|c|}{ Verification cohort } \\
\hline & & $\mathrm{EH}$ & NT \\
\hline N & 11 & 275 & 264 \\
\hline Men: Women (n (\%)) & $7: 4$ & 140 (50.9):135 (49.1) & $127(48.1): 137$ (51.9) \\
\hline Caucasian: Hispanic (n (\%)) & $11: 0$ & 132 (48.0):143 (52.0) & 97 (36.7):167 (53.3) \\
\hline \multicolumn{4}{|l|}{ Age (years, $n(\%)$ ) } \\
\hline 0-19 & - & - & $1(0.4)$ \\
\hline $20-29$ & $1(9.1)$ & - & $10(3.8)$ \\
\hline 30-39 & $1(9.1)$ & $7(2.5)$ & $11(4.2)$ \\
\hline $40-49$ & $4(36.4)$ & $26(9.5)$ & $38(14.4)$ \\
\hline $50-59$ & $3(27.3)$ & $70(25.5)$ & $48(18.2)$ \\
\hline $60-69$ & $2(18.2)$ & $73(26.5)$ & $66(25.0)$ \\
\hline $70-79$ & - & $59(21.5)$ & $51(19.3)$ \\
\hline $80-89$ & - & $34(12.4)$ & $33(12.5)$ \\
\hline $90-99$ & - & $5(1.8)$ & $6(2.3)$ \\
\hline $100+$ & - & $1(0.4)$ & - \\
\hline Mean (SD) & $46.9(11.9)$ & $64.9(13.2)$ & $62.3(16.4)$ \\
\hline \multicolumn{4}{|l|}{ Body mass index (BMI) } \\
\hline Baseline $^{A}$ & $47.9(6.9)$ & $29.6(6.1)$ & $26.9(4.8)$ \\
\hline Post surgery ${ }^{B}$ & $36.0(6.7)$ & - & - \\
\hline$p: A$ vs. B & $6.21 \mathrm{E}-09$ & - & - \\
\hline \multicolumn{4}{|l|}{ Diastolic blood pressure } \\
\hline Baseline $^{A}$ & $91.6(12.8)$ & N/A & N/A \\
\hline Post surgery ${ }^{B}$ & $82.7(7.2)$ & - & - \\
\hline$p: A$ vs. B & $1.48 \mathrm{E}-02$ & - & - \\
\hline \multicolumn{4}{|l|}{ Systolic blood pressure } \\
\hline Baseline $^{A}$ & $144.5(16.0)$ & N/A & N/A \\
\hline Post surgery ${ }^{B}$ & $125.9(14.7)$ & - & - \\
\hline$p: A$ vs. B & $6.78 \mathrm{E}-03$ & - & - \\
\hline
\end{tabular}

Abbreviations: EH essential hypertension, NT normotensive, RYGB Roux-en-Y gastric bypass, N/A not applicable

${ }^{a}$ This group comprises patients who underwent RYGB surgery. Values at baseline and post-surgery are shown as mean values (SD). $P$-values were calculated by means of pairwise t-tests, contrasting values at baseline and six months after RYGB-surgery ( $p$ : A vs. B). A one-tailed $p$-value $<0.05$ was considered significant

levels (Table 3). Overall, no significant differences in the methylation of the 24 detected $\mathrm{CpG}$ sites were detected before and six months after surgical intervention (Table 2).

The methylation of two CpG sites that correlated with SBP levels in the RYGB cohort are differentially methylated in the confirmative cohort showing $\mathrm{EH}$

Two candidate CpG sites (cg00875989; cg09134341) that we found to be associated with SBP in the RYGB cohort, were significantly hypomethylated in $\mathrm{EH}$ (Table 2). These sites are located in the promoter regions of the genes Euchromatic histone-lysine n-methyltransferase 2 (EHMT2) and ski family transcriptional corepressor 2 (SKOR2), respectively. The results were confirmed in the verification cohort by comparing methylation levels in normotensives and EH by binary logistic regression analysis and were independent of age, BMI, ethnicity and sex (Table 2).

\section{Discussion}

By genome-wide investigating the DNA methylation profile in whole blood of RYGB patients we identified two novel CpG sites that were associated with SBP levels and EH. Our findings show that increased methylation of these probes correlate strongly with significant reduction in SBP levels observed after RYGB surgery. In agreement with this, $\mathrm{EH}$ is associated with significantly lower methylation levels of the identified CpG sites compared to normotensive subjects. These results were derived from two independent study populations encompassing healthy, obese, young, middle-aged and elderly 
Table 2 CpG sites significantly correlated with SBP and EH

\begin{tabular}{|c|c|c|c|c|c|c|c|c|c|c|c|c|}
\hline \multirow[b]{3}{*}{ Gene } & \multirow[b]{3}{*}{ Illumina ID } & \multirow[b]{3}{*}{$\begin{array}{l}\text { Distance to } \\
\text { TSS }\end{array}$} & \multicolumn{6}{|c|}{ Discovery group ${ }^{\mathrm{a}}(n=11)$} & \multicolumn{4}{|c|}{ Verification cohort ${ }^{\mathrm{b}}(n=539)$} \\
\hline & & & \multicolumn{3}{|c|}{ \% DNA Methylation (SD) } & \multicolumn{3}{|c|}{$\begin{array}{c}\text { Spearman's rank correlation } \\
\text { coefficients (SBP) } \\
\end{array}$} & \multirow{2}{*}{$\begin{array}{l}\text { \% DNA Methylation } \\
\text { (SD) }\end{array}$} & \multicolumn{3}{|c|}{$\begin{array}{c}\text { Binary logistic regressions } \\
(\mathrm{EH})\end{array}$} \\
\hline & & & Baseline $^{A}$ & After surgery ${ }^{B}$ & p A vs. B & Coef. & $p$ & $p$ (Bonf.) & & $\operatorname{Exp}(B)$ & $p$ & $\overline{p \text { (Bonf.) }}$ \\
\hline MCCC1 & cg00161968 & 27 & $5.36(1.1)$ & $5.9(1.7)$ & ns & 0.93 & $<10 \mathrm{E}-16$ & $<10 \mathrm{E}-16$ & $7.5(4.3)$ & - & ns & ns \\
\hline SKOR2 & cg00875989 & 1500 & $33.2(1.7)$ & 33.6 (3.6) & ns & $(0.92)$ & $<10 \mathrm{E}-16$ & $<10 \mathrm{E}-16$ & $37.5(4.2)$ & 0.92 & 1.16E-03 & $2.79 \mathrm{E}-02$ \\
\hline VGLL3 & cg06251539 & 375 & $9.3(2.4)$ & $8.7(1.88)$ & ns & 0.92 & $<10 \mathrm{E}-16$ & $<10 \mathrm{E}-16$ & $14.5(3.1)$ & - & ns & ns \\
\hline WSB1 & cg08706258 & 125 & $4.3(1.1)$ & $4.2(0.7)$ & ns & 0.93 & $<10 \mathrm{E}-16$ & $<10 \mathrm{E}-16$ & $4.8(0.8)$ & - & ns & ns \\
\hline EHMT2 & cg09134341 & $(1332)$ & $91.0(2.8)$ & $91.8(1.2)$ & ns & $(0.92)$ & $<10 \mathrm{E}-16$ & $<10 \mathrm{E}-16$ & $89.3(1.9)$ & 0.84 & $9.58 \mathrm{E}-04$ & $2.30 \mathrm{E}-02$ \\
\hline TCF12 & $\operatorname{cg} 10146710$ & 1132 & $2.4(0.5)$ & $2.4(0.4)$ & ns & 0.92 & $<10 \mathrm{E}-16$ & $<10 \mathrm{E}-16$ & $2.3(1.0)$ & - & ns & ns \\
\hline MAP2K4 & cg10596925 & (242) & $12.1(3.4)$ & $13.0(2.0)$ & ns & 0.95 & $<10 \mathrm{E}-16$ & $<10 \mathrm{E}-16$ & $10.7(2.9)$ & 0.94 & $4.98 \mathrm{E}-02$ & ns \\
\hline FAM54A & cg10640093 & (196) & $3.8(1.0)$ & $4.1(0.7)$ & ns & 0.94 & $<10 \mathrm{E}-16$ & $<10 \mathrm{E}-16$ & $3.0(0.7)$ & - & ns & ns \\
\hline SORBS1 & $\operatorname{cg} 12360759$ & 598 & $7.4(1.7)$ & $7.4(1.0)$ & ns & 0.92 & $<10 \mathrm{E}-16$ & $<10 \mathrm{E}-16$ & $9.1(1.3$ & 0.82 & $6.33 \mathrm{E}-03$ & ns \\
\hline DDX55 & cg15612682 & 232 & $6.0(1.5)$ & $6.1(0.8)$ & ns & 0.93 & $<10 \mathrm{E}-16$ & $<10 \mathrm{E}-16$ & $5.6(1.0)$ & - & ns & ns \\
\hline ATXN1L & cg16076930 & 380 & $19.0(2.8)$ & 20.5 (5.1) & ns & 0.94 & $<10 \mathrm{E}-16$ & $<10 \mathrm{E}-16$ & $21.1(2.8)$ & - & ns & ns \\
\hline EFEMP1 & $\operatorname{cg} 16118212$ & 227 & $12.7(4.4)$ & $10.7(2.9)$ & ns & 0.92 & $<10 \mathrm{E}-16$ & $<10 \mathrm{E}-16$ & $17.3(6.2)$ & - & ns & ns \\
\hline CRKL & cg16500810 & (197) & $1.5(0.3)$ & $1.6(0.9)$ & ns & 0.92 & $<10 \mathrm{E}-16$ & $<10 \mathrm{E}-16$ & $0.6(0.6)$ & - & ns & ns \\
\hline STEAP3 & $\operatorname{cg} 18643762$ & (211) & $2.9(1.0)$ & $3.4(1.0)$ & ns & $(0.93)$ & $<10 \mathrm{E}-16$ & $<10 \mathrm{E}-16$ & $1.7(1.3)$ & - & ns & ns \\
\hline CPEB4 & cg20841073 & 648 & $10.7(2.2)$ & $10.3(2.7)$ & ns & 0.93 & $<10 \mathrm{E}-16$ & $<10 \mathrm{E}-16$ & $5.2(2.0)$ & 0.85 & 2.37E-03 & ns \\
\hline RHOBTB2 & cg21344124 & 302 & $3.2(0.7)$ & $2.8(1.1)$ & ns & 0.92 & $<10 \mathrm{E}-16$ & $<10 \mathrm{E}-16$ & $2.2(0.7)$ & 1.34 & 1.96E-02 & ns \\
\hline ATXN1 & cg21996137 & (185) & $2.0(0.7)$ & $1.8(0.6)$ & ns & 0.92 & $<10 \mathrm{E}-16$ & $<10 \mathrm{E}-16$ & $1.6(0.7)$ & 1.42 & $1.60 \mathrm{E}-02$ & ns \\
\hline TSSK1B & cg22011370 & 525 & 89.9 (2.6) & 89.9 (2.9) & ns & (0.95) & $<10 \mathrm{E}-16$ & $<10 \mathrm{E}-16$ & 90.6 (3.3) & - & ns & ns \\
\hline SPPL $2 A$ & cg22295383 & (104) & $5.2(1.3)$ & $4.9(1.1)$ & ns & 0.94 & $<10 \mathrm{E}-16$ & $<10 \mathrm{E}-16$ & $4.2(1.3)$ & - & ns & ns \\
\hline BHLHA9 & cg23945265 & 1003 & $45.4(4.2)$ & 46.9 (3.2) & ns & $(0.92)$ & $<10 \mathrm{E}-16$ & $<10 \mathrm{E}-16$ & $45.7(5.2)$ & 0.96 & $2.58 \mathrm{E}-02$ & ns \\
\hline CHCHD5 & cg25521086 & (58) & $4.0(0.8)$ & $3.8(0.7)$ & ns & 0.95 & $<10 \mathrm{E}-16$ & $<10 \mathrm{E}-16$ & $3.1(1.2)$ & - & ns & ns \\
\hline NRTN & cg25544164 & 342 & $99.0(0.3)$ & $9.1(0.2)$ & ns & 0.94 & $<10 \mathrm{E}-16$ & $<10 \mathrm{E}-16$ & $99.1(0.5)$ & - & ns & ns \\
\hline
\end{tabular}

Abbreviations: $E H$ essential hypertension, $\operatorname{Exp(B)}$ log odds ratio, $n s$ not significant, $R Y G B$ Roux-en-Y gastric bypass, SBP systolic blood pressure, SD standard deviation, TSS transcription start site

${ }^{a}$ This group comprises patients who underwent RYGB surgery. ${ }^{\mathrm{b}}$ Results were derived from binary logistic regressions of EH (yes/no) to \% CpG site methylation, age, BMl, ethnicity and sex. Log odds ratios and $p$-values refer to the CpG site in focus. $P$-values for DNA methylation levels were calculated by paired $\mathrm{t}$-tests, contrasting methylation levels at baseline and six months after RYGB-surgery ( $p$ : A vs. B). GWAS results for SBP were calculated by genome-wide analyses of Spearman's rank correlation coefficients using algorithm AS 89 and an Edgeworth series approximation. Unless specified as SD, parenthesis "( $)$ " implies negative numbers

subjects, respectively. The observations suggest that the identified epigenetic risk sites are associated with hypertension and cardiovascular disease across different ages and weight groups.

To our knowledge, this is the first study investigating epigenome-wide associations of whole blood methylation patterns and SBP levels in a longitudinal manner in a RYGB cohort. By observing RYGB patients before and after intervention, we were able to take the dynamic quality of BP and associated methylation changes into account. Thus, our study allows more specifically elucidating BP associated methylation changes as compared to e.g. a case-control study set up, where the likelihood of confounding due to interindividual variance and e.g. (long-time) HT induced secondary methylation changes is much higher. By choosing a genome-wide study approach using the Illumina $450 \mathrm{k}$ methylation chip, it was possible to comprehensively investigate $124199 \mathrm{CpG}$ sites with regard to changes in methylation patterns in a hypothesis-free and thereby unbiased manner. The two CpG sites did not change significantly in their methylation state after RYGB surgery, providing further grist to the mill that their association to SBP is not limited exclusively to this intervention but rather attributable to the general population.

Euchromatic histone-lysine n-methyltransferase 2 (EHMT2; also termed G9a) is a protein lysine histone methyltransferase (PKMT), encoded by EHMT2 and expressed in high levels in peripheral blood leukocytes [26]. G9a is responsible for the majority of dimethylation 
Table 3 CpG site associations to age, BMI, DBP and glucose

\begin{tabular}{|c|c|c|c|c|c|}
\hline \multicolumn{6}{|c|}{ Discovery cohort $^{a}(n=11)$} \\
\hline \multirow[b]{2}{*}{ Gene } & \multirow[b]{2}{*}{ Illumina ID } & \multicolumn{4}{|c|}{ Spearman's rank correlation coefficients } \\
\hline & & $p$ (Age) & $p(\mathrm{BMI})$ & $p$ (DBP) & $p$ (Glucose) \\
\hline MCCC1 & cg00161968 & ns & ns & ns & ns \\
\hline SKOR2 & cg00875989 & ns & ns & ns & ns \\
\hline VGLL3 & cg06251539 & ns & ns & ns & ns \\
\hline WSB1 & cg08706258 & ns & ns & ns & ns \\
\hline EHMT2 & cg09134341 & ns & ns & ns & ns \\
\hline TCF12 & $\operatorname{cg} 10146710$ & ns & ns & ns & $2.81 \mathrm{E}-02$ \\
\hline MAP2K4 & cg10596925 & ns & ns & ns & ns \\
\hline FAM54A & cg10640093 & ns & ns & ns & ns \\
\hline SORBS1 & $\operatorname{cg} 12360759$ & ns & ns & ns & ns \\
\hline DDX55 & cg15612682 & ns & ns & ns & ns \\
\hline ATXN1L & cg16076930 & ns & ns & ns & ns \\
\hline EFEMP1 & cg16118212 & ns & ns & ns & ns \\
\hline CRKL & cg16500810 & ns & ns & ns & $2.08 \mathrm{E}-02$ \\
\hline STEAP3 & cg18643762 & ns & ns & ns & ns \\
\hline CPEB4 & cg20841073 & ns & ns & ns & ns \\
\hline RHOBTB2 & cg21344124 & ns & ns & ns & ns \\
\hline ATXN1 & cg21996137 & ns & ns & ns & ns \\
\hline TSSK1B & cg22011370 & ns & ns & ns & 4.04E-02 \\
\hline SPPL2A & cg22295383 & ns & ns & ns & ns \\
\hline BHLHA9 & cg23945265 & ns & ns & ns & $4.78 \mathrm{E}-02$ \\
\hline CHCHD5 & cg25521086 & ns & ns & ns & ns \\
\hline NRTN & cg25544164 & ns & ns & ns & ns \\
\hline
\end{tabular}

Abbreviations: $D B$ diastolic blood pressure, $n s$, not significant, $R Y G B$, Roux-en- $Y$ gastric bypass, SBP systolic blood pressure

${ }^{a}$ Cohort comprises patients who underwent RYGB surgery. $P$-values were derived by analyses of Spearman's rank correlation coefficients using algorithm AS 89 and an Edgeworth series approximation. For the variables DBP, BMI and fasting plasma glucose, percent change in methylation states were correlated with percent change in these variables, measured before and six months after RYGB surgery. For the variable age, percent change in methylation states measured before and six months after RYGB surgery was correlated with age.

of histone $\mathrm{H} 3$ at lysine 9 (H3K9me2), a major repressive histone methylation mark in euchromatin [27]. Cells lacking G9a display a drastic reduction in this modification [27]. Lehnertz et al. identified a critical role for G9a in T helper cell differentiation in vitro and in vivo. Notably, expression of interleukin 17 (IL-17) was dysregulated in T helper cells in the absence of G9a or in the presence of a specific G9a methyltransferase inhibitor, which is associated with a loss of H3K9me2 at the IL-17 locus. G9a is thus a critical component in the inhibition of IL-17 expression [28]. Several recent studies confirmed the concept that cytokines produced by $\mathrm{T}$ cells and other inflammatory cells contribute to hypertension [29-35]. Harrison et al. identified IL-17 as a novel proinflammatory cytokine that contributes to the development of hypertension. Notably, Harrison et al. found that mice lacking IL-17 do not sustain hypertension after treatment with the hypertensive stimulus angiotensin II [36]. Furthermore, in contrast to the effects seen in wild-type mice, IL-17-/- mice do not exhibit an increase in superoxide production and there is no reduction in endothelialdependent vasodilatation after treatment with angiotensin II [36]. IL-17 further promotes chemotaxis of other proinflammatory cells by stimulating release of chemokines [37, 38]. In agreement with this, Harrison et al. also noted a reduction in vascular accumulation of leukocytes in IL-17 -/- mice treated with angiotensin II [36]. Thus, IL-17 may influence the vascular pathophysiology of hypertension by its direct effects - increasing superoxide production and reducing endothelial-dependent vasodilatation and by guiding other pro-inflammatory cells to the perivascular tissue. Further studies are needed to confirm the role of IL-17 in human $\mathrm{EH}$, and to investigate whether the observed methylation changes in G9a reflect gene expression levels, H3K9me2 activity and IL-17 expression levels. The global role of G9a leaves of course space for additional regulatory functions in HT by, for example, incorporating also effects on the induction of Th2 lineagespecific cytokines interleukin 4, interleukin 5 and interleukin 14 [27] and the cellular response to stress by inhibition of interleukin 6 and interleukin 8 transcription [39]. Nevertheless, in light of these recent scientific advances in the study of hypertension, we suggest that G9a putatively contributes to the pathophysiology of $\mathrm{EH}$ by influencing IL-17 expression (Fig. 1).

SKI family transcriptional co-repressor 2 (SKOR2), also termed functional SMAD-suppressing 235 element on chromosome 18 (FUSSEL18) [40] or Corl2 [41], was first described by Arndt et al. and 236 identified as a novel homolog to the SKI family of transcriptional corepressors [40]. While little is known about its role in human leukocytes, cancer studies have identified this protein as a potential tumor suppressor in head and neck squamous cell carcinomas [42]. Further research is needed before any speculation as to the functional relevance of SKOR2 in EH can be made.

Our study is limited by the fact that BP was measured only once in a sitting position in the discovery cohort, which putatively causes a higher variance of the outcome variable than expected with repeated and averaged measurements. We took the most important confounders available, such as age, gender, BMI and ethnicity, into consideration, on the association analysis between methylation and $\mathrm{EH}$. However, potential confounding factors e.g. dietary patterns, comorbidities or intake of any antihypertensive medication may be able to induce changes in methylation patterns as well. Furthermore, our study pertained to promoter associated CpG sites in DNA methylation in whole blood largely consisting of leukocytes, which are composed of different cell populations with unique 


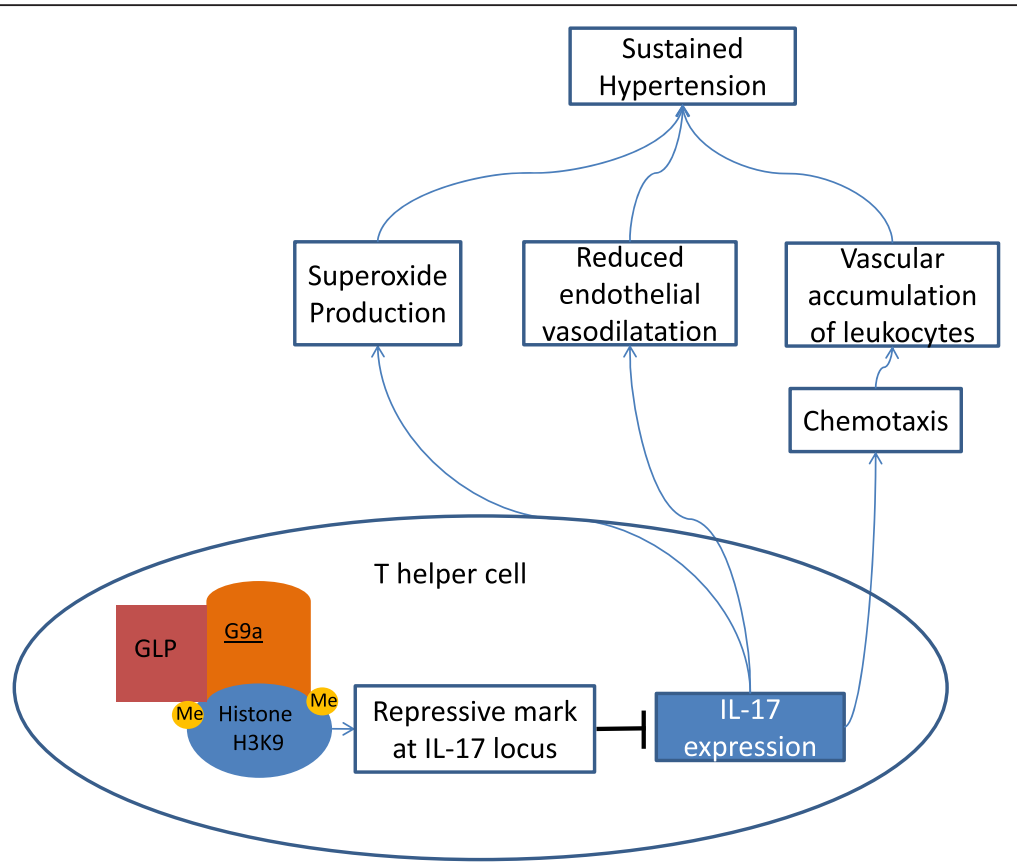

Fig. 1 The methyltransferase G9a in cooperation with its homologue G9a-like protein (GLP) is responsible for the majority of dimethylation of H3K9me2. Cells that lack G9a (or GLP) display a drastic reduction in this modification [26]. H3K9me2 acts as a major repressive histone methylation mark in euchromatin, that affects also the interleukin 17 gene locus, resulting in inhibition of IL-17 expression [27]. Harrison et al. demonstrated that mice lacking IL-17 do not develop sustained hypertension after treatment with angiotensin II. In contrast to wild-type mice, IL-17-/- mice do not exhibit an increase in superoxide production and there is no reduction in endothelial-dependent vasodilatation. Furthermore, there was a reduction in vascular accumulation of leukocytes in IL-17-/- mice, probably due to a reduction of chemotaxis normally induced by IL-17 [35]

epigenetic profiles [43]. Studies indicate that changes in leukocyte composition may induce a significant variability in the DNA methylation pattern observed in the peripheral blood [43]. Koestler et al. provided a list of the top 50 differentially methylated CpG sites observed in six important leukocyte subtypes [44]. None of the newly detected CpG sites belonged to the described group of CpG sites, which supports the reliability of our results. Furthermore, we confirmed our findings in more than 539 individuals in a case-control study, making confounding effects on our results due to changes in leukocyte composition unlikely.

\section{Conclusions}

In summary we were able to identify two novel $\mathrm{CpG}$ loci associated with $\mathrm{EH}$ that have not yet been described in context to HT. Our findings may contribute to a further understanding of the epigenetic regulatory mechanisms behind the development of this disease. Based on our findings we suggest that EHMT2 may contribute to the vascular pathophysiology of $\mathrm{EH}$ by influencing IL-17 expression in T helper cells. Further studies are needed to confirm the role of IL-17 in human $\mathrm{EH}$, and to investigate whether the observed methylation changes in G9a reflect gene expression levels, H3K9me2 activity and IL-17 expression levels.
The global role of G9a leaves space for additional regulatory functions in HT.

\section{Competing interests}

The authors declare that they have no competing interests.

\section{Authors' contributions}

AEB carried out the design of the study, performed the statistical analysis and drafted the manuscript. JM participated in the statistical analysis and review of the manuscript. SV participated in the statistical analysis and review of the manuscript. WW revised the manuscript. BS conceived of the study, and participated in its design and coordination and helped to revise the manuscript. $\mathrm{KZ}$ provided essential data to the study and helped review the manuscript. HS participated in the design of the study, as well as drafting and reviewing of the manuscript. All authors read and approved the final manuscript.

\section{Acknowledgments}

We thank all of our patients that provided the blood samples and clinical information. All authors critically revised the manuscript for important intellectual content and contributed to writing the manuscript. All authors had full access to all of the data and take responsibility for the integrity and accuracy of the data analysis. Work from HB is supported by the Swedish Research Council. JM is supported by the Swedish National Foundation for Medical Research (SSMF).

\section{Author details}

'Department of Neuroscience, Functional Pharmacology, Uppsala University, BMC, Box 59375124 Uppsala, Sweden. ${ }^{2}$ Institute for Genomic Medicine, University of California, San Diego, CA 92093, USA. ${ }^{3}$ eSwiss Medical and Surgical Center, St Gallen, Switzerland.

Received: 29 April 2015 Accepted: 1 April 2016

Published online: 22 April 2016 


\section{References}

1. Kearney PM, Whelton M, Reynolds K, Muntner P, Whelton PK, He J. Global burden of hypertension: analysis of worldwide data. Lancet. 2004;365(9455): 217-33.

2. Shih PAB, O'Conner DT. Hereditary determinants of human hypertension strategies in the setting of genetic complexity. Hypertension. 2008;51(6): 1456-64.

3. Udali S, Guarini P, Moruzzi S, Choi SW, Friso S. Cardiovascular epigenetics: from DNA methylation to microRNAs. Mol Aspects Med. 2013;34(4):883-901.

4. Friso S, Pizollo F, Choi SW, Guarini P, Castagna A, Ravagnani V, et al. Epigenetic control of 11 beta-hydroxysteroid dehydrogenase 2 gene promoter is related to human hypertension. Atherosclerosis. 2008;199(2):323-7.

5. Smolarek I, Wyszko E, Barciszewska AM, Nowak S, Gawronska I, Jablecka A, et al. Global DNA methylation changes in blood of patients with essential hypertension. Med Sci Monit. 2010;16(3):CR149-55.

6. Zhang LN, Liu PP, Wang LY, Yuan F, Xu LT, Xin YF, et al. Lower ADD1 gene promoter DNA methylation increases the risk of essential hypertension. PLoS One. 2013;8(5):e63455

7. Dornfeld LP, Maxwell MH, Waks AU, Schroth P, Tuck ML. Obesity and hypertension - long-term effects of weight reduction on blood pressure. Int J Obes (Lond). 1985:9(6):381-9.

8. Reisin E, Frohlich ED. Effects of weight-reduction on arterial-pressure. J Chronic Dis. 1982;35(12):887-91.

9. Lutz TA, Bueter M. Physiological mechanisms behind Roux-en-Y gastric bypass surgery. Dig Surg. 2014;31(1):13-24.

10. Buchwald $H$, Avidor $Y$, Braunwald $E$, Jensen MD, Pories W, Fahrbach $K$, et al. Bariatric surgery: a systematic review and meta-analysis. JAMA. 2004;292(14): 1724-37.

11. Fenske WK, Dubb S, Bueter M, Seyfried F, Patel K, Tam FWK, et al. Effect of bariatric surgery-induced weight loss on renal and systematic inflammation and blood pressure: a 12-month prospective study. Surg Obes Relat Dis. 2013:9(4):559-68.

12. Chalmers J, MacMahon S, Mancia G, Whitworth J, Beilin L, Hansson L, et al. 1999 World Health Organization International Society of Hypertension quidelines for the management of hypertension. J Hypertens. 1999;17(2):151-83.

13. Wang $X$, Snieder H. Familiar aggregation of blood pressure. In: Flynn JT, Ingelfinger JR, Portman RJ, editors. Clinical Hypertension and Vascular Diseases: Pediatric Hypertension. 2010; 2nd ed: Totowa Humana Press Inc. 241-258A

14. Reckelhoff JF. Gender differences in the regulation of blood pressure. Hypertension. 2001:37(5):1199-208.

15. Margolis KL, Piller LB, Forde CE, Henriquez MA, Cushman WC, Einhorn PT, et al. Blood pressure control in Hispanics in the antihypertensive and lipid-lowering treatment to prevent heart attack trial. Hypertension. 2007;50(5):854-61.

16. Savoia C, Schiffrin EL. Inflammation in hypertension. Curr Opin Nephrol Hypertens. 2006;15(2):152-8.

17. Nilsson E, Ernst B, Voisin S, Almen MS, Benedict C, Mwinyi J, et al. Roux-En Y Gastric Bypass Surgery Induces Genome-Wide Promoter-Specific Changes in DNA Methylation in Whole Blood of Obese Patients. PLoS One. 2015;10(2): e0115186

18. Fried M, Hainer $V$, Basdevant A, Buchwald $H$, Deitel M, Finer N, et al. Interdisciplinary European guidelines on surgery of severe obesity. Int $J$ Obes (Lond). 2008;1(1):52-9.

19. Hannum G, Guinney J, Zhao L, Zhang L, Hughes G, Sadda S, et al. Genomewide methylation profiles reveal quantitative views of human aging rates. Mol Cell. 2013:49(2):359-67.

20. Sambrook J, Fritsch E.F, Maniatis T. Molecular Cloning A Laboratory Manual Second Edition Vols. 12 and 3. Cold Spring Harbor Lab Press. 1989; Vol. 1 p. XXXIX + Pagination Varies. Vol. 2 p. XXXIII + Pagination Varies. Vol. 3 p. XXXII + Pagination Varies.

21. Price M, Cotton M, Lam L, Farre P, Emberly E, Brown C, et al. Additional annotation enhances potential for biologically-relevant analysis of the Illuminalnfinium HumanMethylation450 BeadChip array. Epigenetics Chromatin. 2013;6(1):4.

22. Hsieh CL. Dependency of transcriptional repression on CpG methylation density. Mol Cell Biol. 1994;14(8):5487-94.

23. Wagner JR, Busche $\mathrm{S}, \mathrm{Ge} B$, Kwan T, Pastinen T, Blanchette $M$. The relationship between DNA methylation, genetic and expression inter-individual variation in untransformed human fibroblasts. Genome Biol, 2014:15:R37.

24. Leek JT, Johnson WE, Parker HS, Jaffe AE, Storey JD. The sva package for removing batch effects and other unwanted variation in high-throughput experiments. Bioinformatics. 2012;28(6):882-3.
25. Hochberg Y. A sharper bonferroni procedure for multiple tests of significance Biometrika. 1988;75(4):800-2.

26. Brown SE, Campbell RD, Sanderson CM. Novel NG36/G9a gene products encoded within the human and mouse MHC class III regions. Mamm Genome. 2001;12(12):916-24.

27. Tachibana M, Sugimoto K, Nozaki M, Ueda J, Ohta T, et al. G9a histone methyltransferase plays a dominant role in euchromatic histone $\mathrm{H} 3$ lysine 9 methylation and is essential for early embryogenesis. Genes Dev. 2002; 16(14):1779-91.

28. Lehnertz B, Northrop JP, Antignano F, Burrows K, Hadidi S, et al. Activating and inhibitory functions for the histone lysine methyltransferase G9a in T helper cell differentiation and function. J Exp Med. 2010;207(5):915-22.

29. Tran LT, MacLeod KM, McNeill JH. Chronic etanercept treatment prevents the development of hypertension in fructose-fed rats. Mol Cell Biochem. 2009:330:219-28.

30. Guzik TJ, Hoch NE, Brown KA, McCann LA, Rahman A, et al. Role of the T cell in the genesis of angiotensin II induced hypertension and vascular dysfunction. J Exp Med. 2007;204:2449-60.

31. Venegas-Pont M, Manigrasso MB, Grifoni SC, LaMarca BB, Maric C, et al. Tumor necrosis factor-alpha antagonist etanercept decreases blood pressure and protects the kidney in a mouse model of systemic lupus erythematosus. Hypertension. 2010;56:643-9.

32. Elmarakby AA, Quigley JE, Imig JD, Pollock JS, Pollock DM. TNF-alpha inhibition reduces renal injury in DOCA-salt hypertensive rats. Am J Physiol Regul Integr Comp Physiol. 2008;294:R76-83.

33. Muller DN, Shagdarsuren E, Park JK, Dechend R, Mervaala E, et al. Immunosuppressive treatment protects against angiotensin I-induced renal damage. Am J Pathol. 2008;161:1679-93.

34. Schrader LI, Kinzenbaw DA, Johnson AW, Faraci FM, Didion SP. IL-6 deficiency protects against angiotensin II induced endothelial dysfunction and hypertrophy. Arterioscler Thromb Vasc Biol. 2007;27:2576-81.

35. Brands MW, Banes-Berceli AK, Inscho EW, Al-Azawi H, Allen AJ, et al. Interleukin 6 knockout prevents angiotensin II hypertension: role of renal vasoconstriction and janus kinase 2/signal transducer and activator of transcription 3 activation. Hypertension. 2010;56:879-84.

36. Harrison DG, Guzik TJ, Lob HE, Madhur MS, Marvar PJ, et al. Inflammation, immunity, and hypertension. Hypertension. 2011;57(2):132-40.

37. Hartupee J, Liu C, Novotny M, Li X, Hamilton T. IL-17 enhances chemokine gene expression through mRNA stabilization. J Immunol. 2007;179:4135-41.

38. Kao CY, Huang F, Chen Y, Thai P, Wachi S, et al. Up-regulation of CC chemokine ligand 20 expression in human airway epithelium by IL-17 through JAK-independent but MEK/NG-kappaB-dependent signaling pathway. J Immunol. 2005;175:6676-85.

39. Takahashi A, Imai Y, Yamakoshi K, Kuninaka S, Ohtani N, et al. DNA damage signaling triggers degradation of histone methyltransferases through APC/ C(Cdh1) in senescent cells. Mol Cell. 2012:45(1):123-31.

40. Arndt S, Poser I, Schubert T, Moser M, Bosserhoff AK. Cloning and functional characterization of a new Ski homolog, Fussel-18, specifically expressed in neuronal tissues. Lab Invest. 2005;85(11):1330-41.

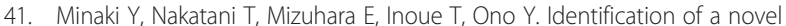
transcriptional corepressor, Corl2, as a cerebella Purkinje cell-selective marker. Gene Expr Patterns. 2008;8(6):418-23.

42. Bennet KL, Lee W, Lamarre E, Zhang XT, Seth R, Scharpf J, et al. HPV statusindependent association of alcohol and tobacco exposure or prior radiation therapy with promoter methylation of FUSSEL 18, EBF3, IRX1, and SEPT9, but not SLC5A8, in head and neck squamous cell carcinomas. Genes Chromosomes Cancer. 2010:49(4):319-26.

43. Reinius LE, Acevedo N, Joerink M, Pershagen G, Dahlen SE, Greco D, et al. Differential DNA methylation in purified human blood cells: implications focr cell lineage and studies on disease susceptibility. PLoS One. 2012;7(7): e41361.

44. Koestler DC, Marsit CJ, Christensen BC, Accomando W, Langevin SM, Houseman EA, et al. Peripheral Blood Immune Cell Methylation Profiles Are Associated with Nonhematopoietic Cancers. Cancer Epidemiol Biomarkers Prev. 2012 21(8):1293-302. 